\title{
Immunization Under Threat During Lebanon's Economic Collapse: A Call for Building on the Lebanese Public System To Avert Yet Another Looming Crisis
}

\author{
Randa Hamadeh ${ }^{1}$, Ola Kdouh ${ }^{2}$, Rawan Hammoud ${ }^{1, *}$ and Jennifer Leaning ${ }^{3}$ \\ ${ }^{1}$ Global Health Team of Experts, Primary Healthcare Department, Ministry of Public Health, Lebanon \\ ${ }^{2}$ Global Health Team of Experts, Ministry of Public Health, Lebanon \\ ${ }^{3}$ Harvard François Xavier Bagnoud Center for Health and Human Rights, Boston, USA
}

\begin{abstract}
Lebanon has been dealing with protracted crises and consecutive existential shocks for decades [1]. Most recently, the triple blow of a severe economic crisis, the Beirut Port Explosion and a global pandemic has wreaked havoc across the country that had already been burdened by the Syrian refugee crisis [2]. In under two years, the Lebanese Lira lost $90 \%$ of its value with dire consequences on the availability of basic services and supplies [3]. The crippling effects of the COVID-19 pandemic have only exacerbated the crisis, placing the healthcare system under extreme stress and hampering imports of medical supplies and essential vaccines. The complex result is a severe shortage of essential vaccines in the private sector and a marked reduction in demand for immunizations on the part of the population.
\end{abstract}

Here we call for a paradigm shift in the procurement and administration of essential vaccines in Lebanon to avoid a national public health catastrophe.

The private sector, which is estimated to vaccinate $45 \%$ of all children in Lebanon, has until now enjoyed a special arrangement with the government and pharmaceutical companies, whereby they would impose a $200 \%$ markup on vaccine costs for patients seeking vaccinations from their offices. In 2018, in order to enhance the access of the population to immunization, the Ministry of Public Health (MOPH) collaborated with the private sector and created a mechanism through which pediatricians could administer vaccines procured by the expanded program on Immunization (EPI) in their clinics provided they did not charge for the vaccine. Fewer than $1 \%$ of pediatricians in Lebanon responded and collaborated at the time, as they were used to their special arrangement. Now, with the economic collapse, the private sector cannot purchase vaccines and are advising families to delay immunizations in their private offices. Yet vaccinations continue to be available for free in the public sector. The EPI under the MOPH currently provides essential vaccines to 800 primary healthcare centers, dispensaries and vaccination outlets across Lebanon [4]. These vaccines are purchased annually by the MOPH through pooled procurement with
UNICEF and administered free of charge to any child residing in Lebanon regardless of nationality.

The demand side for vaccines has also constricted during the consecutive lockdowns and pandemic scares. In line with reports from across the globe, a recent study found that the utilization of immunization services in Lebanon decreased by $31 \%$ at the national level with a utilization decline of $46.9 \%$ in the private sector for the same services [5]. Even as the country lifted lockdowns, the escalating transportation costs have posed a new geographical access barrier for vulnerable populations. A drop in essential vaccine coverage is something Lebanon cannot afford. Lebanon has been battling with vaccine preventable communicable diseases for decades made only harder by the Syrian refugee crisis. Lebanon experiences recurrent measles outbreaks every 5 years and is struggling to maintain the zero Polio status. The low vaccination rates of the past two years as a result of quarantine measures, transportation barriers and shortage of vaccines in the private sector have created large pockets of unvaccinated children. While no measles outbreaks were reported in Lebanon in 2020 and 2021 so far, the situation is expected to quickly change as the restrictions are lifted, children return to their schools and surveillance systems recover from the COVID-19 strain.

While the EPI with its current funding can absorb the increased demand in the short term if collaboration with

*Corresponding author: Rawan Hammoud, Ministry of Public Health, Department of Primary Healthcare, Beirut, Lebanon, Tel: +165-0305-0341

Accepted: December 09, 2021

Published online: December 11, 2021

Citation: Hamadeh R, Kdouh O, Hammoud R, et al. (2021) Immunization Under Threat During Lebanon's Economic Collapse: A Call for Building on the Lebanese Public System To Avert Yet Another Looming Crisis. Arch Community Med 4(1):34-35 
the private sector is successful, a major expansion in the program capacities is definitely warranted. The recent economic collapse and the inability of the private sector to import vaccines have highlighted the enormous gaps in the previous model, where extremely liberal vaccine import laws prioritized private sector profit over community access and the public good. This model has failed in the face of the economic crisis. However, in the midst of crisis, there is opportunity for change. As such, we call on the Lebanese government to mandate the pooled procurement of all essential vaccines for all children in Lebanon, negating the need for parallel imports by the private sector. We call upon the pediatricians in the private sector to prioritize the health of their patients ahead of their own profits and sign up to be part of the EPI vaccine administration program. We also ask the international community to continue support Lebanon in these trying times and ensure sustainability of funding for routine vaccination services and campaigns to avoid a major public health disaster. As the world tries to recover from the COVID-19 pandemic, it is our duty to protect vulnerable populations and not neglect other public health threats and successful interventions; routine immunization.

\section{Declarations}

Ethics approval and consent to participate: Not applicable

Consent for publication: Not applicable
Availability of supporting data: All articles used as reference were available in open source.

Competing interests: The authors declare that they have no competing interests.

Funding: No funding was received to write or publish this Commentary.

\section{Authors' contributions}

All authors participated equally in the review of the literature, in the discussions, in structuring the commentary, and contributed to the writing of all sections of this paper.

Acknowledgements: Not Applicable

\section{References}

1. DeJong J (2020) The challenges of a public health approach to COVID-19 amid crises in Lebanon. Middle East Report Online.

2. Hamadeh RS, Kdouh O, Hammoud R, et al. (2021) Working short and working long: can primary healthcare be protected as a public good in Lebanon today? Conflict and health 15: 1-9.

3. OCHA (2021) Emergency Response Plan Lebanon.

4. Hemadeh R, Kdouh O, Hammoud R, et al. (2020) The primary healthcare network in Lebanon: A national facility assessment. East Mediterr Health J 26: 700-707.

5. Mansour Z, Arab J, Said R, et al. (2021) Impact of COVID-19 pandemic on the utilization of routine immunization services in Lebanon. PLoS One 16.

DOI: $10.36959 / 547 / 648$ 